\title{
Space interferometry beyond exoplanetology: Can interdisciplinary collaboration contribute to the future of this technique?
}

\author{
Pavel Gabor ${ }^{1}$ \\ ${ }^{1}$ Vatican Observatory, Vatican City \\ email: p.gabor@jesuit.cz
}

\begin{abstract}
Although a formation-flying space interferometer designed for exoplanet spectroscopy is feasible in principle, the novelty and cost of such an instrument is likely to remain daunting unless the scientific benefits of this technology are demonstrated by intermediary, precursor missions. Such instruments would represent intermediary steps in the real-life testing of the technology, and therefore, by the very reason of being intermediary, they may not have the resolving or collecting power needed for the study of the objects where biomarkers could be hoped to be detected, i.e., exo-Earths in the habitable zone of their stars. This paper examines the potential applications of such intermediary instruments. The direct line of thought focuses on exoplanetology (gas giants, protoplanetary discs, Neptunes, super-Earths, etc.); what we would like to stimulate is an exercise in lateral thinking, looking at what might an intermediary interferometric mission contribute to other fields of astrophysical research (galaxies, supernova precursors, planetary nebulae, molecular clouds, etc.). The paper raises the question of collaboration with astrophysicists studying areas other than exoplanets and its potential gains for the future of space interferometry.
\end{abstract}

Keywords. instrumentation: interferometers, techniques: high angular resolution, space vehicles: instruments

\section{Nulling Interferometry in Space}

Since its inception in 1993, the proposed Darwin nulling interferometer (Léger et al. 1996) together with its US counterpart TPFI, has been an important factor in a number of instrumental studies. The science case, as currently presented, is this: The study of extrasolar planets, for the moment, relies on indirect methods. At some point, however, the field will only be able to advance if efficient direct imaging (including spectroscopy) becomes available. The feasibility of the concept has been demonstrated quite satisfactorily by Peters et al. (2010) and Martin \& Booth (2010). In the eyes of many it was the clear choice for a flagship space mission (cf. Liseau 2010). The funding agencies, however, have not selected this mission for implementation so far. Consequently, if realised, the launch of Darwin/TPFI is highly unlikely before 2025 .

Apart from a nulling interferometer, other approaches have been under development (the most advanced ones being an occulter and an internal coronograph). Since 2008, roadmapping has been under way both in Europe (ExoPlanetary Roadmap Advisory Team; Blue Dots Initiative; Coudé Du Foresto et al. 2010), and in the US (Exoplanet Analysis Committee; Exoplanet Task Force; Traub et al. 2010), seeking consensus as to the best strategy in future instrumentation, with special attention to a flagship space mission. The outcome of the process remains unclear while a sense of urgency is growing.

A major issue appears to be a certain reluctance towards space interferometry which is present to varying degree not only among decision makers but also in the scientific 
community at large, among astrophysicists in general as well as among exoplanetologists and astrobiologists. This attitude can be easily understood, considering that

- even ground-based visible/IR interferometry is very difficult to implement,

- few ground-breaking science results have been obtained using this technique,

- nulling is considerably more demanding than constructive interferometry, and

- implementing this method in space adds another degree of difficulty to the enterprise.

\section{A Scientifically Valuable Precursor}

In order to overcome this ambivalence, interferometry needs to demonstrate its potential. Schneider (2009) called for a "scientifically valuable precursor". Such an accomplishment could indeed bring about a change of mentality. How to make such a precursor valuable not only technologically but also scientifically?

Historically, the space interferometer community focuses on circumstellar environements . This may be creating an unnecessary dilemma for the precursor. On the one hand, a precursor, by definition, does not achieve the performance of the flagship mission, and therefore - in exoplanetology - it is unlikely to produce major discoveries, nor even can it do cutting-edge science, e.g., as a pathfinder. On the other hand, since the flagship mission's application is exoplanetology, other fields are passed over even when planning a precursor. But beyond exoplanetology it could prove itself "scientifically valuable".

A wider scope of science targets may benefit the cause of a precursor immensely.

(a) First, by simple opening more possibilities of achieving inspiring results, and,

(b) second, by involving a larger portion of the astrophysical community as a whole. This applies to space precursors, but also to ground-based (and airborne) instruments.

\section{Broadening Horizons}

Is there anything a space interferometer could contribute to the field of star formation? In addition to protostellar disks, envelopes, jets, and debris disks, could it discover any new features (let us recall that polar jets were an unexpected phenomenon)? What about molecular clouds or planetary nebulae, and other stellar remnants? Is there anything it could contribute to the study of active galactic nuclei? Or perhaps of quasars? (3C 273 with its jet could be an interesting targets because a detailed map may allow to infer the physical nature of the quasar; Uchiyama et al. 2006.) And what about microquasars, black-hole accretion disks, supernova precursors, and other interacting binary systems?

\section{References}

Coudé Du Foresto, V. \& Blue Dots 2010, in V. Coudé Du Foresto, D. M. Gelino, \& I. Ribas (eds.), ASP Conf. Ser., 430, 15

Léger, A., Mariotti, J.-M., Mennesson, B., Ollivier, M., Puget, J.-L., Rouan, D., \& Schneider, J. 1996, Icarus, 123, 249

Liseau, R. 2010 in V. Coudé Du Foresto, D. M. Gelino, \& I. Ribas (eds.), ASP Conf. Ser., 430, 219

Martin, S. R. \& Booth, A. J. 2010, A\&A, 520, A96

Peters, R. D., Lay, O. P., \& Lawson, P. R. 2010, PASP, 122, 85

Schneider, J. 2009, ArXiv, 0906.0068

Traub, W. A., Lawson, P. R., Unwin, S. C., Muterspaugh, M. W., Soummer, R., Danchi, W. C., Hinz, P., Gaudi, B. S., Torres, G., Deming, D., Lazio, J., \& Dressler, A. 2010, in V. Coudé Du Foresto, D. M. Gelino, \& I. Ribas (eds.), ASP Conf. Ser., 430, 21

Uchiyama, Y., Urry, C. M., Cheung, C. C., Jester, S., Van Duyne, J., Coppi, P., Sambruna, R. M., Takahashi, T., Tavecchio, F., \& Maraschi, L. 2006, ApJ, 648, 910 